\title{
Usefulness of Lipoprotein (a) for Predicting Progression of Non-Culprit Coronary Lesions After Acute Myocardial Infarction
}

Hiroki Ikenaga, MD; Masaharu Ishihara, MD, PhD; Ichiro Inoue, MD, PhD; Takuji Kawagoe, MD, PhD; Yuji Shimatani, MD; Fumiharu Miura, MD, PhD; Yasuharu Nakama, MD; Kazuoki Dai, MD; Takayuki Otani, MD; Kentaro Ejiri, MD; Nozomu Oda, MD; Masayuki Nakamura, MD; Takashi Miki, MD

\begin{abstract}
Background: The serum lipoprotein (a) [Lp(a)] level is genetically determined and remains consistent during a person's life. Previous cohort studies have reported that subjects with a high $L p(a)$ level are at high risk of cardiac events.

Methods and Results: This study consisted of 410 patients who underwent primary percutaneous coronary intervention within $24 \mathrm{~h}$ of the onset of acute myocardial infarction (AMI). Lp(a) was measured 1 week after AMI and patients were divided into 2 groups based: high $L p(a)$ group $(>40 \mathrm{mg} / \mathrm{dl}, \mathrm{n}=95)$ and low $L p(a)$ group $(\leq 40 \mathrm{mg} / \mathrm{dl}, \mathrm{n}=$ 315). A major adverse cardiac event (MACE) was defined as cardiac death, myocardial infarction and/or revascularization for new lesions. The incidence of MACE during 5 years was significantly higher in the high Lp(a) group than in the low $L p(a)$ group ( $34.7 \%$ vs. $16.5 \%, P<0.001)$. This difference was primarily driven by a higher incidence of new lesions requiring revascularization in the high $L p(a)$ group $(31.6 \%$ vs. $15.2 \%, P<0.001)$. Multivariate analysis showed that $L p(a)$ was an independent predictor for MACE (odds ratio [OR] 1.64, 95\% confidence interval [Cl] $1.31-2.06, \mathrm{P}<0.001)$ and revascularization of a new lesion (OR 1.61, 95\% Cl 1.32-2.13, $\mathrm{P}<0.001)$.
\end{abstract}

Conclusions: $\mathrm{Lp}(\mathrm{a})$ levels could predict the progression of the non-culprit coronary lesions after AMI. (Circ $J$ 2011; 75: 2847-2852)

Key Words: Acute myocardial infarction; Lipoprotein (a); Prognosis; Revascularization

$\mathbf{L}$ ipoprotein (a) $[\mathrm{Lp}(\mathrm{a})]$ consists of a low-density lipoprotein (LDL) particle with its apolipoprotein B-100 component linked by a disulfide bridge to apolipoprotein (a). The serum $\mathrm{Lp}$ (a) level is genetically determined, remains consistent within an individual over several years and is unaltered by diet or exercise. ${ }^{1} \mathrm{Lp}(\mathrm{a})$ has structural similarity to plasminogen, causing competition with plasminogen activators of humans. ${ }^{2-4}$ Experimental studies have reported that $L p(a)$ enters the arterial intima and promotes thrombosis, inflammation, and foam cell formation. ${ }^{5,6}$ Several clinical studies have demonstrated that elevated levels of serum $\mathrm{Lp}(\mathrm{a})$ are associated with the progression of coronary artery disease and an increased risk of cardiovascular events, independent of other conventional risk factors. ${ }^{2,4,7}$ However, there are few data on the prognostic utility of $\mathrm{Lp}(\mathrm{a})$ in patients with acute myocardial infarction (AMI) who undergo primary percutaneous coronary intervention (PCI). This study was undertaken to assess whether the $\mathrm{Lp}$ (a) level could predict cardiac events after primary PCI for AMI.

\section{Methods}

From September 1999 to April 2007 at Hiroshima City Hospital, 894 patients with AMI underwent primary PCI within $24 \mathrm{~h}$ of the onset of chest pain. Among these patients, this study investigated 410 in whom $L p(a)$ was measured 1 week after AMI. AMI was diagnosed by chest pain consistent with ongoing myocardial ischemia persisting longer than $30 \mathrm{~min}$ with concomitant ECG changes. Serum creatine kinase (CK) was measured every $3 \mathrm{~h}$ and the peak $\mathrm{CK}$ value had to be more than twice the normal upper limit. Emergency coronary angiography and primary PCI was performed as previously reported. ${ }^{7}$ Selective coronary angiography was performed in multiple projections before the initiation of reperfusion therapy. Immediately after diagnostic angiography, primary PCI was performed with conventional balloon angioplasty or stent.

One week after AMI, a blood sample was obtained after overnight fasting. Levels of $\mathrm{Lp}(\mathrm{a})$, apolipoprotein A1, apolipoprotein $\mathrm{B}$, apolipoprotein $\mathrm{E}$, remnant-like particles cholesterol,

Received April 6, 2011; revised manuscript received July 29, 2011; accepted August 2, 2011; released online September 13, 2011 Time for primary review: 25 days

Department of Cardiology, Hiroshima City Hospital, Hiroshima, Japan

Mailing address: Masaharu Ishihara, MD, PhD, FACC, Department of Cardiology, Hiroshima City Hospital, 7-33 Moto-machi, Naka-ku, Hiroshima 730-8518, Japan. E-mail: ishifami@fb3.so-net.ne.jp

ISSN-1346-9843 doi:10.1253/circj.CJ-11-0365

All rights are reserved to the Japanese Circulation Society. For permissions, please e-mail: cj@j-circ.or.jp 


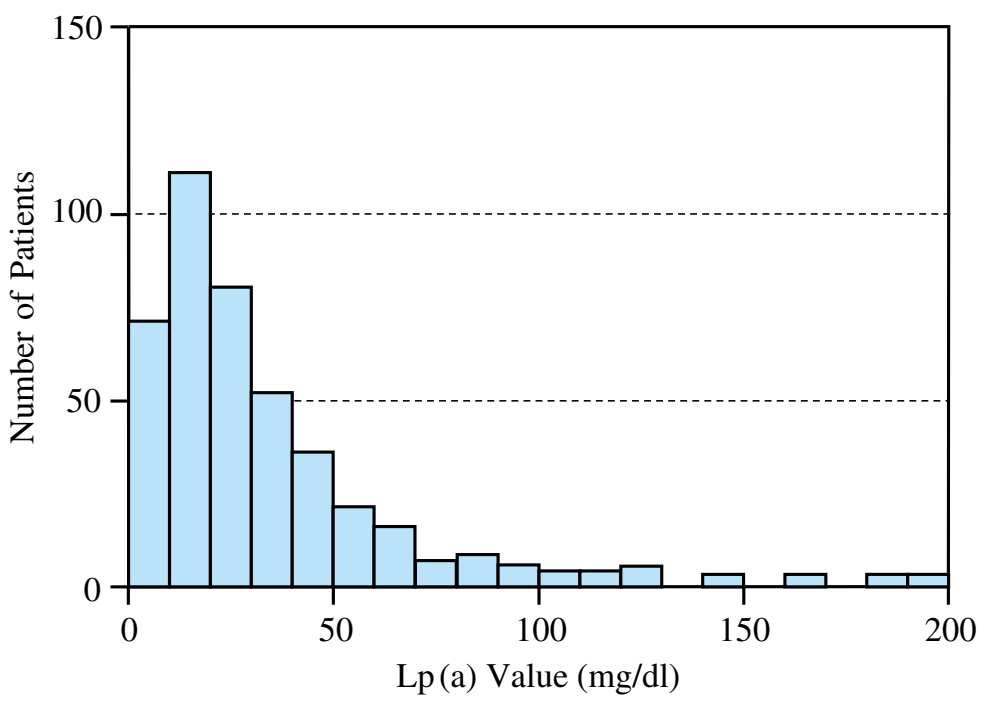

Figure 1. Histogram of lipoprotein (a) [Lp(a)] value.

Table 1. Baseline Characteristics

\begin{tabular}{lccc} 
& Low $\mathbf{L p}(\mathbf{a})(\mathbf{n}=\mathbf{3 1 5})$ & High Lp(a) (n=95) & P value \\
Age (years) & $63 \pm 12$ & $64 \pm 11$ & 0.32 \\
Male & $259(82 \%)$ & $79(83 \%)$ & 0.83 \\
Hypertension & $147(46 \%)$ & $43(45 \%)$ & 0.80 \\
Diabetes mellitus & $70(22 \%)$ & $22(23 \%)$ & 0.85 \\
Current smoker & $166(53 \%)$ & $51(54 \%)$ & 0.87 \\
Time to angiography (h) & $4.3 \pm 4.6$ & $4.5 \pm 4.3$ & 0.64 \\
Killip class 3-4 & $29(9.2 \%)$ & $7(7.3 \%)$ & 0.57 \\
Initial TIMI 0-1 flow & $239(76 \%)$ & $70(74 \%)$ & 0.67 \\
Collateral circulation & $135(45 \%)$ & $36(39 \%)$ & 0.34 \\
Anterior MI & $148(47 \%)$ & $48(51 \%)$ & 0.54 \\
MVD & $94(30 \%)$ & $29(31 \%)$ & 0.90 \\
Final TIMI 3 & $293(93 \%)$ & $90(94 \%)$ & 0.61 \\
eGFR <60 mI. min-1.1.73m-2 & $111(35 \%)$ & $36(38 \%)$ & 0.66 \\
Medications at discharge & & & \\
$\quad$ Statins & $106(34 \%)$ & $32(34 \%)$ & 0.99 \\
ACEI/ARB & $191(61 \%)$ & $60(63 \%)$ & 0.76 \\
$\beta$-blocker & $100(32 \%)$ & $29(31 \%)$ & 0.81 \\
\hline
\end{tabular}

Lp(a), lipoprotein (a); TIMI, Thrombolysis In Myocardial Infarction; eGFR, estimated glomerular filtration rate; ACEI/ ARB, angiotensin-converting enzyme inhibitor/angiotensin receptor blocker; MI, myocardial infarction; MVD, multivessel disease.

Table 2. Serum Lipid and Lp(a) Levels

$\mathrm{Lp}(\mathrm{a})(\mathrm{mg} / \mathrm{dl})$

Total cholesterol $(\mathrm{mg} / \mathrm{dl})$

Triglycerides ( $\mathrm{mg} / \mathrm{dl}$ )

HDL-cholesterol $(\mathrm{mg} / \mathrm{dl})$

LDL-cholesterol ( $\mathrm{mg} / \mathrm{dl})$

LDL/HDL-cholesterol ratio

Apolipoprotein A1 (mg/dl)

Apolipoprotein B (mg/dl)

Apolipoprotein $\mathrm{E}(\mathrm{mg} / \mathrm{dl})$

apoB/apoA1 ratio

RLP-cholesterol (mg/dl)

Low $\operatorname{Lp}(a)(n=315)$
$18.5 \pm 1.0$
$190.2 \pm 2.1$
$138.0 \pm 3.8$
$39.2 \pm 0.7$
$123.4 \pm 1.9$
$3.41 \pm 0.07$
$108.1 \pm 3.1$
$104.5 \pm 1.4$
$4.3 \pm 0.1$
$1.02 \pm 0.02$
$5.0 \pm 0.2$

\section{High Lp(a) ( $\mathrm{n}=95)$}

$67.2 \pm 1.8$

$196.5 \pm 3.7$

$142.0 \pm 6.9$

$38.2 \pm 1.2$

$129.9 \pm 3.3$

$3.69 \pm 0.14$

$103.9 \pm 5.6$

$116.3 \pm 2.5$

$4.4 \pm 0.1$

$1.15 \pm 0.03$

$5.1 \pm 0.3$
$P$ value

$<0.001$

0.14

0.61

0.45

0.09

0.08

0.51

$<0.001$

0.24

$<0.001$

0.89

Lp(a), lipoprotein (a); HDL, high-density lipoprotein; LDL, low-density lipoprotein; apoB/apoA1, apolipoprotein B/apolipoprotein $A 1 ; R L P$, remnant-like lipoprotein particle. 


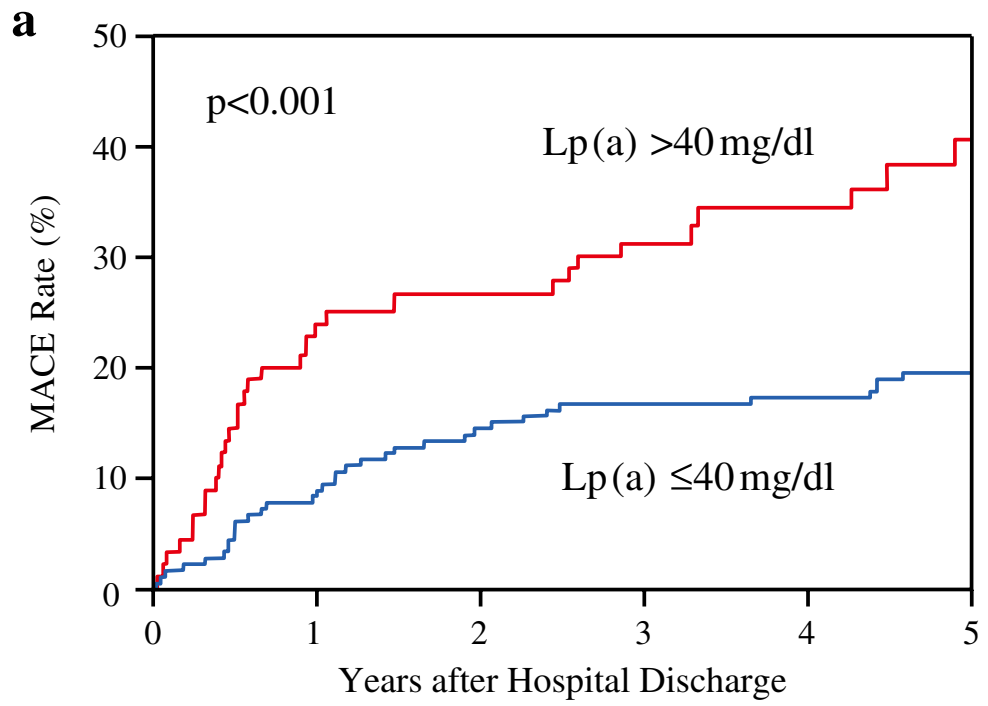

b

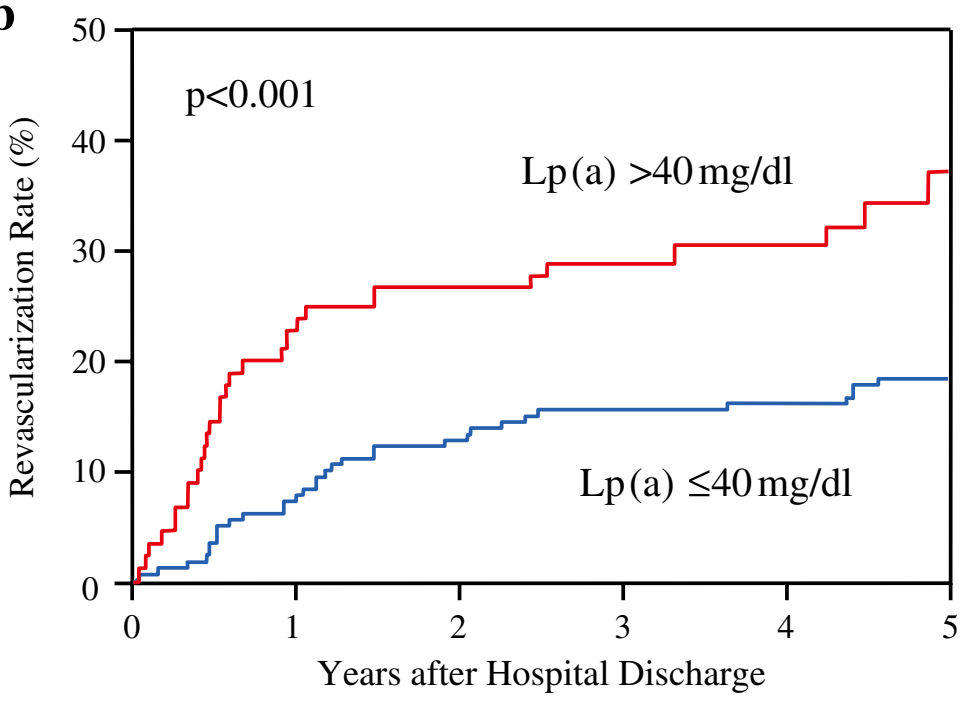

Figure 2. (a) MACE after hospital discharge in patients with high or low $L p(a)$. (b) Revascularization for new lesions in patients with high or low $L p(a)$. Lp(a), lipoprotein (a); MACE, major adverse cardiac event.

total cholesterol, LDL-cholesterol, high-density lipoprotein (HDL) cholesterol, triglycerides, glucose, insulin, hemoglobin $A_{1 c}$ and creatinine were measured. Serum Lp(a) was measured by turbidimetric immunoassay system (BML, Tokyo, Japan). The estimated glomerular filtration rate was calculated using the Japanese equation for estimating glomerular filtration rate from serum creatinine, and chronic kidney disease (CKD) was considered to be present if it was $<60 \mathrm{ml} \cdot \mathrm{min}^{-1} \cdot 1.73 \mathrm{~m}^{-2}$. Multivessel disease (MVD) was defined as $\geq 75 \%$ stenosis in 1 or more vessels remote from the infarcted artery. Informed consent was given by each patient and the study was approved by the ethical committee at Hiroshima City Hospital.

Patients were classified into 2 groups based on Lp(a) level: high Lp(a) group ( $>40 \mathrm{mg} / \mathrm{dl}: \mathrm{n}=95)$ and low $\mathrm{Lp}$ (a) group ( $\leq 40 \mathrm{mg} / \mathrm{dl}: \mathrm{n}=315$ ). Because a serum Lp(a) level of $40 \mathrm{mg} / \mathrm{dl}$ was the $75^{\text {th }}$ percentile of this population and $40 \mathrm{mg} / \mathrm{dl}$ was the normal upper limit in this assay, we defined patients with a serum Lp(a) level $>40 \mathrm{mg} / \mathrm{dl}$ as the high Lp(a) group. Clinical follow-up was obtained up to 5 years. Follow-up angiography was encouraged at 6 and 12 months after primary PCI or earlier when clinically indicated. A major adverse cardiac event (MACE) was defined as cardiac death, MI and/or revascular-

\begin{tabular}{|lccc|}
\hline \multicolumn{4}{|c|}{ Table 3. Cofactors of MACE After Hospital Discharge in } \\
Patients With High or Low Lp(a) \\
Cardiac death & Low Lp(a) & High Lp(a) & P value \\
MI & $5(1.6 \%)$ & $5(5.3 \%)$ & 0.06 \\
Non-TLR & $7(2.2 \%)$ & $5(5.3 \%)$ & 0.14 \\
\hline
\end{tabular}

MACE, major adverse cardiac event; non-TLR, non-target lesion revascularization (revascularization for new lesions). Other abbreviations see in Table 1.

ization for new lesions. Revascularization for new lesions was defined as revascularization for $>75 \%$ stenosis for non-target lesion that progressed more than 1 grade according to the classification of the American Heart Association. Cardiac death was defined as death from pump failure, sudden cardiac death or death because of arrhythmia. We also assessed the incidence of target lesion revascularization (TLR), but did not include it in MACE. 


\begin{tabular}{|c|c|c|c|}
\hline & $\chi^{2}$ & OR (95\%Cl) & $P$ value \\
\hline High Lp(a) & 16.96 & $1.64(1.31-2.06)$ & $<0.001$ \\
\hline MVD & 22.76 & 1.75 (1.39-2.19) & $<0.001$ \\
\hline CKD & 7.98 & $1.51(1.13-2.02)$ & 0.005 \\
\hline Age (years) & 0.12 & $1.01(0.98-1.03)$ & 0.73 \\
\hline Male & 0.51 & $0.90(0.66-1.19)$ & 0.47 \\
\hline Hypertension & 1.38 & $1.14(0.91-1.43)$ & 0.24 \\
\hline Diabetes mellitus & $<0.01$ & $1.01(0.77-1.30)$ & 0.93 \\
\hline Current smoker & 0.18 & $0.98(0.75-1.20)$ & 0.67 \\
\hline Prior MI & 1.26 & $0.81(0.53-1.16)$ & 0.26 \\
\hline Killip class $>1$ & 1.33 & $1.22(0.86-1.66)$ & 0.25 \\
\hline Time to angiography (h) & 2.15 & $1.04(0.99-1.11)$ & 0.14 \\
\hline Anterior MI & 2.43 & $1.19(0.96-1.50)$ & 0.12 \\
\hline Initial TIMI 0-1 flow & 1.45 & $0.86(0.68-1.10)$ & 0.23 \\
\hline Final TIMI 3 & 0.33 & $1.16(0.73-2.12)$ & 0.57 \\
\hline
\end{tabular}

OR, odds ratio; $\mathrm{Cl}$, confidence interval; CKD, chronic kidney disease. Other abbreviations see in Tables 1,3.

\begin{tabular}{|lrcc|}
\hline \multicolumn{4}{|c|}{$\begin{array}{l}\text { Table 5. Independent Predictors for Revascularization of } \\
\text { New Lesions }\end{array}$} \\
High Lp(a) & \multicolumn{1}{c|}{$\chi^{2}$} & OR (95\%Cl) & P value \\
MVD & 16.57 & $1.61(1.32-2.13)$ & $<0.001$ \\
CKD & 23.81 & $1.81(1.43-2.30)$ & $<0.001$ \\
Age (years) & 8.02 & $1.54(1.14-2.09)$ & 0.005 \\
Male & 1.00 & $1.01(0.98-1.04)$ & 0.32 \\
Hypertension & 0.26 & $0.92(0.67-1.24)$ & 0.61 \\
Diabetes mellitus & 1.86 & $1.18(0.93-1.49)$ & 0.17 \\
Current smoker & 0.18 & $1.06(0.80-1.37)$ & 0.67 \\
Prior MI & 0.20 & $0.95(0.73-1.21)$ & 0.65 \\
Killip class $>1$ & 0.82 & $0.84(0.55-1.21)$ & 0.36 \\
Time to angiography (h) & 1.18 & $1.22(0.85-1.67)$ & 0.28 \\
Anterior MI & 2.00 & $1.04(0.98-1.11)$ & 0.16 \\
Initial TIMI 0-1 flow & 0.65 & $1.10(0.87-1.39)$ & 0.42 \\
Final TIMI 3 & 0.71 & $0.89(0.69-1.17)$ & 0.40 \\
\hline
\end{tabular}

Abbreviations see in Tables 1,4.

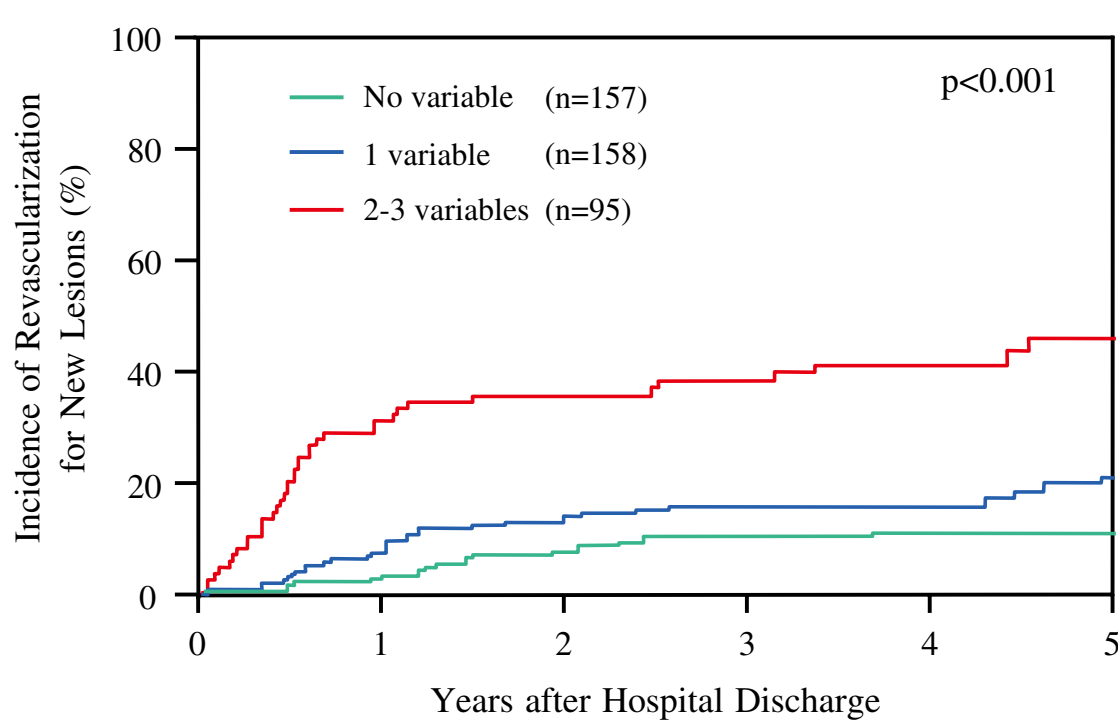

Figure 3. Number of variables and revascularization for new lesions.

\section{Statistical Analysis}

We used standard statistical methods. We tested the significance of difference with $\chi^{2}$ test for categorical variables. Student's t-test was used for continuous variables. Event-free survival curves up to 5 years after AMI were constructed with the Kaplan-Meier method and were compared with the log-rank test. Cox proportional hazard regression was used to identify independent predictors of mortality, adjusting baseline clinical and angiographic variables. We used the JMP statistical package (version 5.0.1 J) for all statistical tests. A significance level of 0.05 was used and 2-tailed tests were applied.

\section{Results}

The mean value of $\mathrm{Lp}(\mathrm{a})$ was $29.7 \pm 26.9 \mathrm{mg} / \mathrm{dl}$, ranging from 1.0 to $190 \mathrm{mg} / \mathrm{dl}$ (Figure 1). Baseline clinical and angiographic characteristics of the study patients are shown in Table 1. There was no significant difference in baseline variables between the high and low Lp(a) groups.
Table 2 shows the laboratory data for the 2 groups. Patients with high Lp(a) had a significantly higher apoliprotein B level and apolipoprotein B/apolipoprotein A1 ratio than patients with low $\mathrm{Lp}(\mathrm{a})$.

Figure 2a shows the 5-year cumulative incidence of MACE, which was significantly higher in patients with high $\mathrm{Lp}(\mathrm{a})$ than in those with low $\mathrm{Lp}$ (a) $(34.7 \%$ vs. $16.5 \%, \mathrm{P}<0.001)$. The incidence of each constituent factor of MACE at 5 years is shown in Table 3. The difference in MACE was primarily driven by a higher incidence of revascularization for new lesion (Figure 2b). Cardiac death, MI and revascularization for new lesions tended to be more frequent in patients with high $\mathrm{Lp}(\mathrm{a})$, but the differences did not reach statistical significance. There was no significant difference in the incidence of TLR $(12.7 \%$ vs. $20.0 \%, \mathrm{P}=0.10$ ).

Multivariate analysis showed that $\mathrm{Lp}(\mathrm{a})$ was an independent predictor for MACE (odds ratio [OR] 1.64, 95\% confidence interval $[\mathrm{CI}] 1.31-2.06, \mathrm{P}<0.001$, Table 4) and revascularization for new lesions (OR 1.61, 95\%CI 1.32-2.13, $\mathrm{P}<0.001$, 
Table 5). Other factors associated with 5-year revascularization for new lesion were MVD and CKD. If the utility of combining these 3 factors (high Lp(a), MVD and CKD), to predict revascularization for new lesions during the 5 years after AMI was assessed, there was a stepwise increase in revascularization for new lesions as the number of factors increased (Figure 3 ).

\section{Discussion}

This study showed that high $\mathrm{Lp}(\mathrm{a})$ is an independent predictor of MACE after AMI in patients treated with primary PCI. It was primarily driven by the more frequent need of revascularization for new lesions in patients with high $\mathrm{Lp}(\mathrm{a})$. There are some mechanisms underlying the development of atherosclerosis. The pathophysiologic role of $L p(a)$ in atherosclerotic disease progression is explained by the accumulation of $\mathrm{Lp}(\mathrm{a})$ in the vessel wall and its ability to promote cholesterol accumulation in macrophages forming foam cells and subsequent fatty streaks ${ }^{3,4}$ and to promote smooth muscle cell proliferation and migration in atherosclerotic lesions by inactivating transforming growth factor- $\beta . .^{5}$ In contrast, the $\mathrm{Lp}$ (a) level was not associated with TLR. Since the introduction of coronary stents, the initial results of primary PCI have dramatically improved, ${ }^{8,9}$ The main cause of TLR is the growth of smooth muscle cells and fibroatheroma, which is thought to be strongly inhibited by the metallic scaffold of the stent. ${ }^{10}$ However, this mechanism of TLR is different from that of non-TLR, so $\mathrm{Lp}$ (a) level was not associated with TLR. In addition, the procedure of primary PCI affects TLR. The use of drug-eluting stent (DES) further reduces TLR. Recently, the ACC/AHA guidelines updated their recommendations that it is reasonable to use DES as an alternative to bare metal stents for primary PCI in AMI patients. Despite the reduction in the MACE rate, however, DES does not improve survival. ${ }^{11}$ It has been reported that, for the patients who survived AMI, long-term outcomes are determined mostly by events attributable to disease progression unrelated to the target lesion treated. Early risk stratification to predict disease progression of non-culprit lesions is, thus, important to improve the outcomes of patients with AMI.

Previous studies have investigated the relation of $\operatorname{Lp}(\mathrm{a})$ to cardiovascular events. ${ }^{12,13}$ Recently, the Emerging Risk Factors Collaboration reported a meta-analysis of 126,634 participants in 36 prospective studies, ${ }^{14}$ which showed that $\mathrm{Lp}(\mathrm{a})$ concentration was independently associated with the risk of coronary heart disease and stroke, but was unrelated to the aggregate of nonvascular mortality. The $\mathrm{Lp}$ (a) concentration was more consistent within individuals over several years than were levels of cholesterol, blood pressure, or other conventional risk factors. ${ }^{15,16}$ In the current study, we measured Lp(a) 1 week after AMI, and showed that a high Lp(a) concentration was associated with a higher incidence of revascularization for new lesions after AMI. The association was independent of conventional risk factors. Multivariate analysis showed that $\mathrm{Lp}(\mathrm{a})$, CKD and MVD, among the conventional risk factors, were independent predictors for MACE and revascularization for new lesions. $\mathrm{Lp}(\mathrm{a})$ is a biomarker that is genetically determined and consistent within individuals. On the other hand, CKD is frequently associated with progressive decline in the glomerular filtration rate, which leads to endstage renal disease and cardiovascular disease. CKD might enhance the activation of both the sympathetic nervous system and the renin-angiotensin-aldosterone system. ${ }^{17}$ So, CKD is a marker that reflects increased levels of inflammatory and arterial sclerosis. In turn, MVD reflects atherosclerosis that is already present. $\mathrm{Lp}(\mathrm{a})$,
CKD and MVD represent different markers of the development of new lesions, and the combination of Lp(a) with MVD and CKD was more useful for early risk stratification in patients with AMI who underwent primary PCI than each factor alone.

Prevention of atherothrombotic events of non-culprit lesions is important to reduce MACE after AMI in patients treated with primary PCI. Several studies have reported that the use of statins with a lower target LDL-cholesterol level slows atherosclerosis progression or reduces coronary plaque volume. ${ }^{18-20}$ However, in this study, statins were prescribed in only onethird of the patients. Aggressive risk management may improve the outcomes of AMI patients with high Lp(a) concentrations. Although apolipoprotein-B100 is a component of Lp(a), contradictory findings have been reported about the effect of statins on the $\mathrm{Lp}$ (a) concentration. Importantly, available data are consistent with the existence of a causal relationship between high $\mathrm{Lp}$ (a) level and cardiac events, and increase the priority for investigating $\operatorname{Lp}(\mathrm{a})$ as a potential therapeutic target. Niacin can reduce the serum Lp(a) level. ${ }^{21,22}$ Recently, the Arterial Biology for the Investigation of the Treatment Effects of Reducing Cholesterol 6-HDL and LDL Treatment Strategies (ARBITE 6-HALTS) trial reported that niacin, when added to simvastatin for patients with low HDL level, decreased the carotid intima-media thickness. ${ }^{23}$ However, the $\mathrm{Lp}$ (a) concentration was not measured and the increased HDL level may be attributable to the benefits obtained by niacin. Further studies are advocated to investigate better therapeutic options for patients with AMI and high Lp(a).

\section{Study Limitations}

This study suffers from the limitations of all retrospective studies. Small sample size is another limitation. In this study, 1 week after AMI, the samples were obtained because almost all patients were discharge within 10 days after AMI. However, it was been reported that most lipoprotein values decrease between 7 and 10 days after the onset of AMI. ${ }^{24}$ By contrast, Slunga et al, in a study of 32 AMI patients, found no clear evidence of $L p(a)$ elevation in the acute phase. ${ }^{25}$ Other reports suggest that the $\mathrm{Lp}(\mathrm{a})$ concentration reaches a peak on the $5^{\text {th }}$ day and then returns to the initial value within 1 week. ${ }^{26}$ Further investigations are necessary to determine the transient increase in serum $\mathrm{Lp}(\mathrm{a})$ concentration after AMI.

\section{Disclosure}

There is no financial support for this study.

\section{References}

1. Seed M, Ayres KL, Humphries SE, Miller GJ. Lipoprotein (a) as a predictor of myocardial infarction in middle-aged men. Am J Med 2001; 110: $22-27$

2. Terres W, Tatsis E, Pfalzer B, Beil FU, Beisiegel U, Hamm CW. Rapid angiographic progression of coronary artery disease in patients with elevated lipoprotein(a). Circulation 1995; 91: 948-950.

3. Liu AC, Lawn RM. Lipoprotein(a) and atherogenesis. Trends Cardiovasc Med 1994; 4: 40-44.

4. Maher VMG, Brown BG. Lipoprotein(a) and coronary heart disease. Curr Opin Lipidol 1995; 6: 229-235.

5. Grainger DJ, Kirschenlohr HL, Metcalfe JC, Weissberg PL, Wade DP, Lawn RM. Proliferation of human smooth muscle cells promoted by lipoprotein(a). Science 1993; 260: 1655-1658.

6. Hartmann M, von Birgelen C, Mintz GS, Stoel MG, Eggebrecht H, Wieneke H, et al. Relation between lipoprotein(a) and fibrinogen and serial intravascular ultrasound plaque progression in left main coronary arteries. J Am Coll Cardiol 2006; 48: 446-452.

7. Desmarais RL, Sarembock IJ, Ayers CR, Vernon SM, Powers ER, Gimple LW. Elevated serum lipoprotein(a) is a risk factor for clinical recurrence after coronary balloon angioplasty. Circulation 1995; 91: $1403-1409$. 
8. Morice MC, Serruys PW, Sousa JE, Fajadet J, Ban Hayashi E, Perin $\mathrm{M}$, et al. A randomized comparison of a sirolimus-eluting stent with a standard stent for coronary revascularization. $N$ Engl J Med 2002; 346: $1773-1780$.

9. Moses JW, Leon MB, Popma JJ, Fitzgerald PJ, Holmes DR, O'Shaughnessy C, et al. Sirolimus-eluting stents versus standard stents in patients with stenosis in a native coronary artery. $N$ Engl $J$ Med 2003; 349: 1315-1323.

10. Morita Y, Himeno H, Yakuwa H, Usui T. Serum lipoprotein(a) level and clinical coronary stenosis progression in patients with myocardial infarction. Circ J 2006; 70: 156-162.

11. Menichelli M, Parma A, Pucci E, Fiorilli R, De Felice F, Nazzaro M, et al. Randomized trial of sirolimus-eluting stent versus bare-metal stent in acute myocardial infarction (SESAMI). J Am Coll Cardiol 2007; 49: 1924-1930.

12. Fujino A, Watanabe T, Kunii H, Yamaguchi N, Yoshinari K, Watanabe Y, et al. Lipoprotein(a) is a potential coronary risk factor. Circ J 2000; 64: $51-56$

13. Igarashi Y, Aizawa Y, Satoh T, Konno T, Ojima K, Aizawa Y. Predictors of adverse long-term outcome in acute myocardial infarction patients undergoing primary percutaneous transluminal coronary angioplasty. Circ J 2003; 67: 605-611.

14. The Emerging Risk Factors Collaboration. Lipoprotein(a) concentration and the risk of coronary heart disease, stroke and nonvascular mortality. JAMA 2009; 302: 412-423.

15. Bennet A, Di Angelantonio E, Erqou S, Eiriksdottir G, Sigurdsson G, Woodward M, et al. Lipoprotein(a) levels and risk of Future coronary heart disease. Arch Intern Med 2008; 168: 598-608.

16. Berg K. A new serum type system in man: The lipoprotein system. Acta Pathol Microbiol Scand 1963; 59: 369-382.

17. Iwanaga Y, Miyazaki S. Heart failure, chronic kidney disease, and biomarkers. Circ J 2010; 74: 1274-1282.

18. Takayama T, Hiro T, Yamagishi M, Daida H, Hirayama A, Saito S, et al. Effect of rosuvastatin on coronary atheroma in stable coronary artery disease: Multicenter coronary atherosclerosis study measuring effects of rosuvastatin using intravascular ultrasound in Japanese subjects (COSMOS). Circ J 2009; 73: 2110-2117.

19. Miyauchi K, Daida H. Clinical significance of intensive lipid-lowering therapy using statins in patients with coronary artery disease: LDL-cholesterol: The lower, the better; is it true for Asians? (Pro) Circ J 2010; 74: 1718-1730.

20. Sakamoto T, Ogawa H. "Just make it lower" as an alternative strategy of lipid-lowering therapy with statins in Japanese patients: LDL-cholesterol: The lower, the better; is it true for Asians? (Con) Circ J 2010; 74: $1731-1741$.

21. McKenney JM, Jones PH, Bays HE, Knopp RH, Kashyap ML, Ruoff $\mathrm{GE}$, et al. Comparative effects on lipid levels of combination therapy with a statin and extended-release niacin or ezetimibe versus a statin alone (the COMPELL study). Atherosclerosis 2007; 192: 432 437.

22. Armstrong VW, Schleef J, Thiery J, Muche R, Schuff-Werner P, Eisenhauer T, et al. Effect of HELP-LDL-apheresis on serum concentrations of human lipoprotein(a): Kinetic analysis of the post-treatment return to baseline levels. Eur J Clin Invest 1989; 19: 235-240.

23. Taylor AJ, Villines TC, Stanek EJ, Devine PJ, Griffen L, Miller M, et al. Extended-release niacin or ezetimibe and carotid intima-media thickness. N Engl J Med 2009; 361: 2113-2122.

24. Maeda S, Abe A, Seishima M, Makino K, Noma A, Kawade M. Transient changes of serum lipoprotein(a) as an acute phase protein. Atherosclerosis 1989; 78: 145-150.

25. Slunga L, Johnson O, Dahlen GH. Change in Lp(a) lipoprotein levels during the treatment of hypercholesterolaemia with simvastatin. Eur J Clin Pharmacol 1992; 43: 369-373.

26. Kawade M, Maeda S, Abe A, Yamashiro M. Alternations in plasma $\mathrm{Lp}$ (a) lipoprotein[Lp(a)] and acute phase proteins after surgical operation (abstract). Clin Chem 1984; 30: 941. 